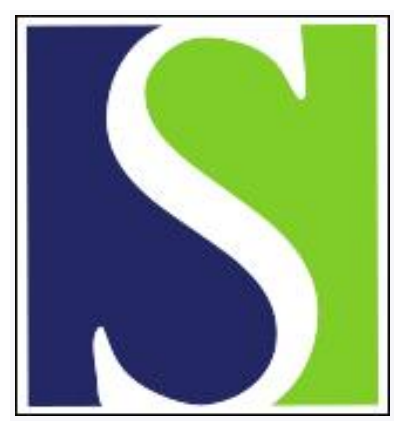

Scand J Work Environ Health 1979;5(2):135-142

https://doi.org/10.5271/sjweh.2659

Issue date: Jun 1979

Conjugation and urinary excretion of toluene and $m$-xylene metabolites in a man

by Riihimäki V

The following articles refer to this text: 1979;5(3):217-231;

$1979 ; 5(3): 232-248$

Key terms: benzoic acid; conjugation; conjugation reaction; hippuric acid; m-methylbenzoic acid; m-methylhippuric acid; m-xylene; $\mathrm{m}$-xylene metabolite; man; toluene; urinary excretion

This article in PubMed: www.ncbi.nlm.nih.gov/pubmed/472684 


\title{
Conjugation and urinary excretion of toluene and m-xylene metabolites in a man
}

\author{
by VESA RIIHIMÄKI, M.D., M.Sc. ${ }^{1}$
}

\begin{abstract}
RIIHIMÄKI, V. Conjugation and urinary excretion of toluene and m-xylene metabolites in a man. Scand. j. work environ. \& health 5 (1979) 135-142. Ingestion of benzoic acid (intermediary metabolite of toluene) by one volunteer subject indicated that the maximum urinary excretion rate of hippuric acid (final metabolite of toluene) was about $190 \mathrm{mmol} / \mathrm{min}$. An almost identical figure for the saturation kinetics of hippuric acid formation has been reported in the literature, and it has been shown to be limited by the availability of glycine for benzoic acid conjugation. The maximum excretory capacity of the kidneys for hippurate is known to be considerably larger than the observed maximum rate of benzoic acid conjugation. In addition the intravenous administration of methylhippuric acid showed that its urinary excretion is rapid, approaching an excretion rate constant of $1.3 \mathrm{~h}^{-1}$. The glycine conjugation of benzoic acid and methylbenzoic acid may well be the ratelimiting step in toluene and, respectively, xylene metabolism. During the inhalation of these solvents, uptake rate may be estimated to equal the full conjugating capacity at a toluene or xylene air concentration of about $780 \mathrm{ppm}$ at rest or of about 270 ppm during moderately heavy work. These data suggest that the body has a relatively large capacity for the conjugation reactions of toluene and xylene metabolism, but the consumption of a large part of easily mobilized glycine may impair the conjugation, and hence excretion, of poorer substrates, e.g., drugs.
\end{abstract}

Key words: benzoic acid, conjugation reactions, hippuric acid, man, m-methylbenzoic acid, m-methylhippuric acid, m-xylene, toluene, urinary excretion.

Several examples of kinetic interactions between drugs and chemicals in the human body are well known, and they may arise from a variety of different kinetic events with different underlying mechanisms (4). As an example, when the biotransformation or excretion capacity of the body is limited for a particular chemical, another competing compound may interact in a significant way.

1 Department of Industrial Hygiene and Toxicology, Institute of Occupational Health, Helsinki, Finland.

Reprint requests to: Dr. Vesa Riihimäki, Institute of Occupational Health, Haartmaninkatu 1, SF-00290 Helsinki 29, Finland.
This phenonenon can be applied to benzoic acid, which is the main oxidized intermediate of toluene metabolism (7). Benzoic acid is, in man, almost completely conjugated with glycine and excreted as hippuric acid via the kidneys (14). The conjugation reaction may become ratelimited by the availability of glycine (1, 14). The mobilization of endogenous glycine was estimated nearly 50 years ago to take place maximally at a rate of 122 $155 \mu \mathrm{mol} / \mathrm{min}$, depending on body size (14), but more recent data indicate that the maximum rate of hippurate formation is about $190 \mu \mathrm{mol} / \mathrm{min}$ (1) and that it can be considerably increased by an additional supply of exogenous glycine. In some spe- 
cies benzoic acid is primarily conjugated with glucuronic acid (3), and even in man increasing doses of benzoic acid, from 6.9 to $69 \mathrm{mmol}$, increase the proportion of glucuronidation from 0.4 to $3.0 \%$ of the total conjugation (16).

Xylene is mainly oxidized to methylbenzoic (toluic) acid, which in turn is conjugated with glycine to produce methylhippuric (toluric) acid and excreted in the urine (18). In rabbit (2) and rat (9) large doses of xylene isomers (5.7-11.3 mmol/ $\mathrm{kg}$ ) have been shown to result also in glucuronide formation, which was greatly dependent on the isomer in question, for $m$-xylene glucuronides constituted up to $13 \%$ of the dose (9). In man low doses of xylenes, absorbed during the inhalation of these compounds at a concentration of 1.9 $\mathrm{mmol} / \mathrm{m}^{3}$ (46 ppm v/v), were not biotransformed to detectable amounts of glucuronide conjugates of methylbenzoic acid (18).

It is well known that the renal excretion of p-aminohippuric acid and hippuric acid takes place almost exclusively via proximal tubular secretion with a transport maximum $\left(\mathrm{T}_{\mathrm{m}}\right)$ on the order of 400 $\mu \mathrm{mol} / \mathrm{min}(20)$. It is very likely that the same transport mechanism secretes even methylhippurate with a rather similar capacity. Quite apparently, there are great similarities between the metabolism and excretion of toluene and xylene, and the correlations between the two compounds in this respect have also been demonstrated in human studies involving inhalation exposures to toluene and xylene both singly and in combination $(10,12,13)$.

In the context of inhalation exposure to toluene and xylene little has been discussed about the capacity of the body to biotransform and excrete these compounds and their metabolites, or about the specific roles of biotransformation and excretion in the elimination kinetics of these compounds. Possible kinetic interactions between toluene and xylene and their metabolites have not been investigated, either. Therefore it was of interest to make some guiding observations on the conjugation of benzoic acid and methylbenzoic acid in one man, as well as on his renal excretion of hippuric acid and methylhippuric acid, both after the administration of the compounds individually and together.

\section{MATERIAL AND METHODS}

\section{Administration of compounds}

For ethical and practical reasons the studies were carried out with only one healthy male research worker. He was 35 years of age and weighed $70 \mathrm{~kg}$. In the single exposures the subject orally ingested one dose of the following one at a time after overnight fasting: $7.4 \mathrm{mmol}$ of m-methylbenzoic acid (Fluka AG, Buchs, Switzerland), $41 \mathrm{mmol}$ of benzoic acid (Merck AG, Darmstadt, Switzerland), 7.8 mmol of m-methylhippuric acid (synthetized at the Department of Organic Chemistry, University of Helsinki and found to be about $99 \%$ pure) and $33.5 \mathrm{mmol}$ of hippuric acid (Fluka AG, Buchs, Switzerland). The test compounds were taken in gelatin capsules.

In the combined exposures the subject ingested, after overnight fasting, (a) 7.4 mmol of methylbenzoic acid followed $2 \mathrm{~h}$ later by $41 \mathrm{mmol}$ benzoic acid and (b) 7.8 mmol of methylhippuric acid followed $1 \mathrm{~h}$ later by $33.5 \mathrm{mmol}$ of hippuric acid. In all experiments the subject did not take any food up to at least $1 \mathrm{~h}$ after the last dose, but he did drink water to maintain sufficient diuresis for the urinary sampling.

On one occasion $0.5 \mathrm{mmol}$ of methylhippuric acid $(5 \mathrm{ml}$ of sterilized solution containing $100 \mathrm{mmol} / 1$ of methylhippuric acid, diluted with $5 \mathrm{ml}$ of physiological saline) was administered in one injection into the cubital vein.

\section{Collection of samples}

After the administration of the test compounds, urine was sampled at 1-h intervals for at least the first $4 \mathrm{~h}$, thereafter at 2-h intervals for the next $8 \mathrm{~h}$. All the urine excreted throughout the first 25$30 \mathrm{~h}$ after the ingestion of the test compounds was collected, stored a few hours at $+5{ }^{\circ} \mathrm{C}$ and then kept at $-20^{\circ} \mathrm{C}$ until analyzed.

\section{Analysis of samples}

Determination of m-methylbenzoic acid in the urine samples was performed with 
Table 1. Urinary excretion of toluene and $\mathrm{m}$-xylene metabolites after oraI intake. $\quad(\mathrm{BA}=$ benzoic acid, $\mathrm{MBA}=$ methylbenzoic acid, $\mathrm{HA}=$ hippuric acid, $\mathrm{MHA}=$ methylhippuric acid)

\begin{tabular}{|c|c|c|c|c|}
\hline \multirow{2}{*}{ Dose } & \multicolumn{2}{|c|}{$\begin{array}{l}\text { Percentage of } \\
\text { dose excreted }\end{array}$} & \multicolumn{2}{|c|}{$\begin{array}{l}\text { Excretion rate } \\
\text { constant b }\left(h^{-1}\right)\end{array}$} \\
\hline & as $\mathrm{HA}$ & as MHA & HA & MHA \\
\hline $41 \mathrm{mmol} \mathrm{BA}$ & $95^{\mathrm{a}}$ & & 1.0 & \\
\hline $7.4 \mathrm{mmol} \mathrm{MBA}$ & & 106 & & 1.0 \\
\hline $7.4 \mathrm{mmol} \mathrm{MBA}+41 \mathrm{mmol} \mathrm{BA}$ & $107^{a}$ & 105 & 1.0 & 1.0 \\
\hline $33.5 \mathrm{mmol} \mathrm{HA}$ & 101 a & & 0.13 & \\
\hline $7.8 \mathrm{mmol}$ MHA & & 107 & & 0.34 \\
\hline $7.8 \mathrm{mmol} \mathrm{MHA}+33.5 \mathrm{mmol} \mathrm{HA}$ & 99 a & 84 & 0.11 & 0.34 \\
\hline
\end{tabular}

a Basal hippurate excretion (mean of two series of determinations) has been subtracted.

b Excretion $5-10 \mathrm{~h}$ after ingestion.

gas chromatography both prior to and after alkaline hydrolysis according to Engström et al. (5). In the benzoic acid analysis the method was slightly modified so that methyl i-butylketone (MIBK) replaced diethylether in the solvent extraction. Silylation was carried out in this solvent and no pyridine was added.

The alkaline hydrolysis used $\mathbf{( 5 . 5}$ $\mathrm{mol} / 1 \mathrm{NaOH}, 110^{\circ} \mathrm{C}$ for $1 \mathrm{~h}$ ) is known to hydrolyze both glycine conjugates and glucuronides $(5,16)$. Therefore, determining benzoic acid and methylbenzoic acid after alkaline treatment provides an assessment of the total conjugates of these compounds. A specific analysis of either glucuronides or glycine conjugates was not made. The coefficient of variation of standard assays was $\pm 4.8 \%$ for hippuric acid and m-methylhippuric acid at a concentration level of $0.5 \mathrm{mmol} / \mathrm{l}$.

\section{RESULTS}

The measured urinary elimination of ingested test compounds was complete in all but one case (table 1). In this particular instance $84 \%$ of the ingested methylhippuric acid was recovered in the urine, and the somewhat slow start of renal excretion (fig. 1) suggested a slow and incomplete absorption from the alimentary tract.

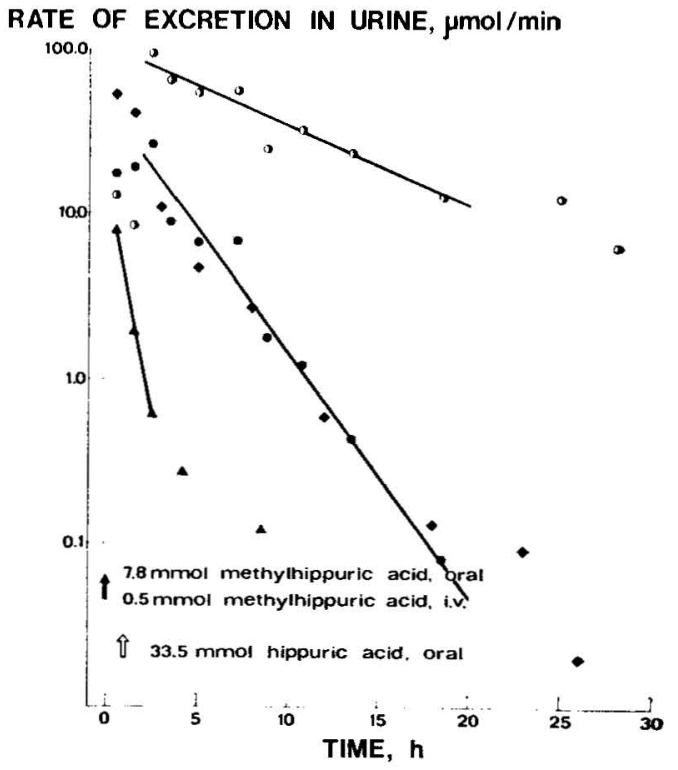

Fig. 1. Urinary excretion of methylhippuric acid after the ingestion of (a) $7.8 \mathrm{mmol}$ of methylhippuric acid ( $\bullet$ ) and (b) $7.8 \mathrm{mmol}$ of methylhippuric acid followed by $33.5 \mathrm{mmol}$ of hippuric acid $1 \mathrm{~h}$ later $(\bullet)$; urinary excretion of hippuric acid in case a (o); and urinary excretion of methylhippuric acid after an intravenous injection of $0.5 \mathrm{mmol}$ of methylhippuric acid (A). Regression lines for the excretion of methylhippuric acid (case a) and hippuric acid (case b) over $2-20$ postingestion hours are shown.

The total recovery achieved for the compounds indicates that no significant metabolites were missed in the assays and that all excretion took place via the kidneys. 
Conjugation and renal excretion of benzoic acid and m-methylbenzoic acid after ingestion of these acids

The excretion of both the benzoic acid and methylbenzoic acid conjugates was rapid for some $4-5 \mathrm{~h}$ after the ingestion of the acids, the excretion rate constants being on the order of $1.0 \mathrm{~h}^{-1}$ (fig. 2). Within $2 \mathrm{~h}$ of the ingestion of $41 \mathrm{mmol}$ of benzoic acid, the urinary excretion of benzoic acid conjugates reached a constant level of $180-198 \mu \mathrm{mol} / \mathrm{min}$ and remained so for $3 \mathrm{~h}$. When the ingestion of methylbenzoic acid $(7.4 \mathrm{mmol})$ was followed by the intake of benzoic acid $(41 \mathrm{mmol}) 2 \mathrm{~h}$ later, the excretion of methylbenzoic acid

\section{RATE OF EXCRETION IN URINE, $\mu \mathrm{mol} / \mathrm{min}$}

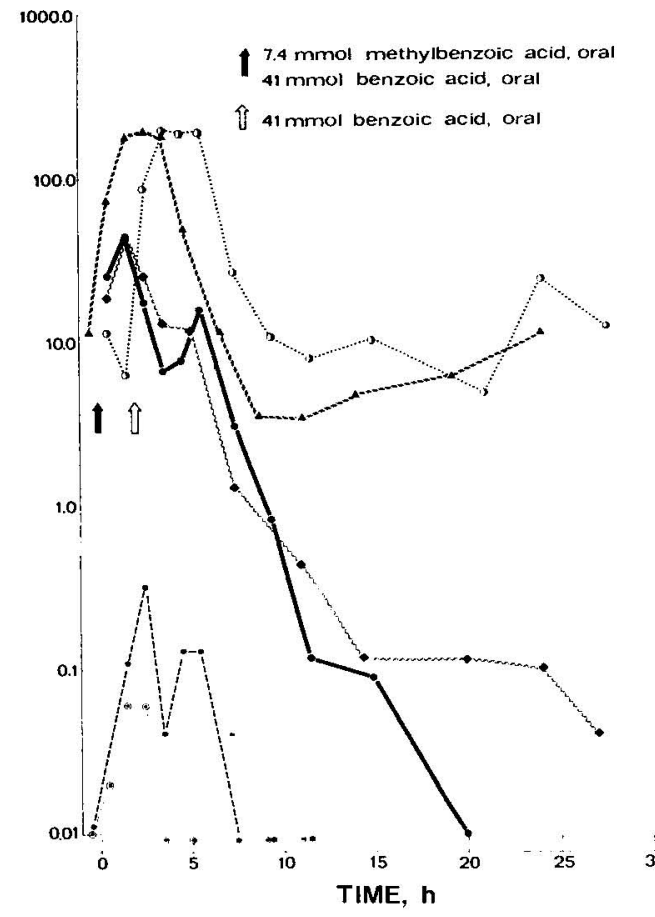

Fig. 2. Urinary excretion of methylbenzoic: acid conjugates (methylhippuric acid) after the ingestion of (a) $7.4 \mathrm{mmol}$ of methylbenzoic acid ( man and (b) $7.4 \mathrm{mmol}$ ) methylbenzoic acid followed by $41 \mathrm{mmol}$ of benzoic acid $2 \mathrm{~h}$ later $(-)$; urinary excretion of senzoic acid conjugates (hippuric acid) after the ingestion of (c) $41 \mathrm{mmol}$ of benzoic acid ( $\Delta=-\boldsymbol{A})$ and (d) $7.4 \mathrm{mmol}$ of methylbenzoic acid followed by $41 \mathrm{mmol}$ of benzoic acid $2 \mathrm{~h}$ later $(0 \cdots 0)$; and urinary excretion of free methylbenzoic acid in case a $(\odot--, \odot)$ and $b$ $(+\cdots+-\infty)$. conjugates seemed to decrease during the concomitant rise of benzoic acid conjugate excretion, only to increase again in spite of the still maximal excretion of benzoic acid conjugates (fig. 2, note the logarithmic ordinate scale). The combined intake of methylbenzoic acid and benzoic acid did not affect the conjugation or excretion of the latter. Fig. 2 also indicates that the excretion of ingested benzoic acid and methylbenzoic acid was nearly complete in about $10 \mathrm{~h}$.

Renal excretion of hippuric acid and $m$-methylhippuric acid after oral and intravenous administration

The urinary excretion rate of methylhippuric acid was halved in about $2 \mathrm{~h}$ (excretion rate constant $\mathrm{k}=0.34 \mathrm{~h}^{-1}$ ), while the corresponding time for hippuric acid was about $6 \mathrm{~h}\left(\mathrm{k}=0.11 \mathrm{~h}^{-1}\right)$ after the ingestion of the respective compounds (fig. 1). The urinary excretion of intravenously injected methylhippuric acid was much more rapid than that of ingested methylhippuric acid, and the excretion rate was halved in about $30 \mathrm{~min}\left(\mathrm{k}=1.3 \mathrm{~h}^{-1}\right.$ ) (fig. 1). Ingestion of $33.5 \mathrm{mmol}$ of hippuric acid $1 \mathrm{~h}$ after $7.8 \mathrm{mmol}$ of methylhippuric acid had been ingested did not affect the renal excretion of the latter (fig. 1).

The differences in the renal excretion of hippuric acid after the ingestion of 41 mmol of benzoic acid and $33.5 \mathrm{mmol}$ of hippuric acid are shown in fig. 3. The excretion of ingested hippuric acid was slow, and nearly $20 \mathrm{~h}$ were needed for complete excretion.

\section{Excretion of free benzoic acid and m-methylbenzoic acid}

Only traces of free benzoic acid and methylbenzoic acid were detected in the urine after the compounds were ingested (total $29-\mathrm{h}$ excretion $0.2-0.8 \%$ of that of the respective conjugates). The highest rates of free methylbenzoic acid excretion followed the combined ingestion of methylbenzoic acid and a large dose of benzoic acid (fig. 2). 
Fig. 3. Urinary excretion of hippuric acid after the ingestion of $41 \mathrm{mmol}$ of benzoic acid $(\Delta-\Delta)$ and $33.5 \mathrm{mmol}$ of hippuric acid ( $\bullet$ ). The basal rates of hippurate excretion on two occasions (mean and range) are also shown (-)). Numbers in parentheses denote rates of diuresis $(\mathrm{ml} / \mathrm{h})$.
RATE OF HIPPURIC ACID EXCRETION IN URINE, $\mu \mathrm{mol} / \mathrm{min}$ 1000.0

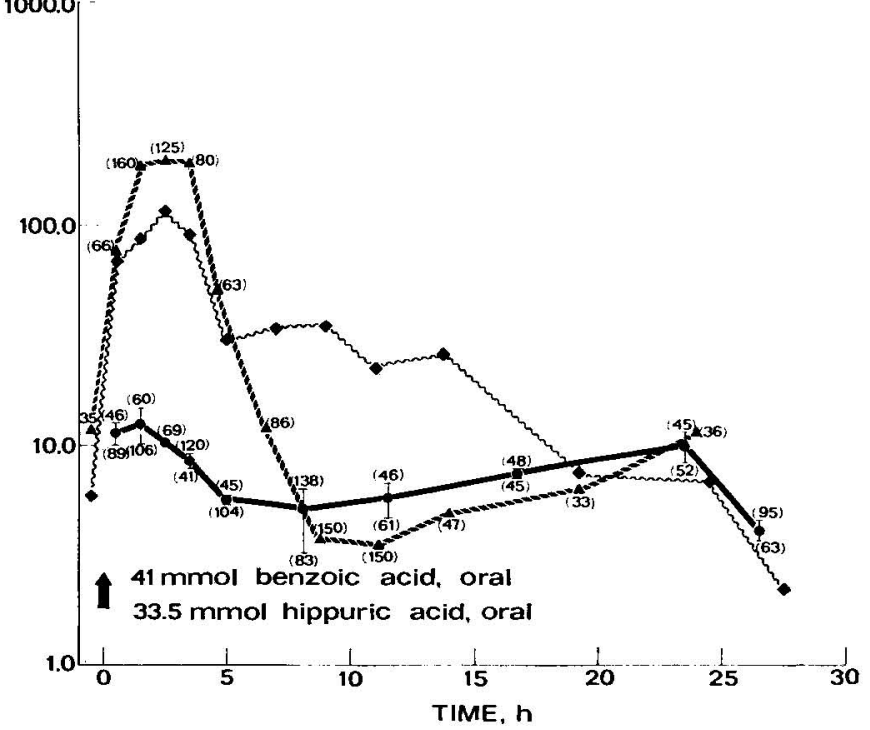

\section{DISCUSSION}

In this investigation the maximum rate of the renal excretion of benzoic acid conjugates (combined glycine and glucuronic acid conjugates) in a 70-kg man after ingestion of the acid was about $190 \mu \mathrm{mol} /$ min. Amsel and Levy (1) obtained almost exactly the same figure for the maximum urinary excretion of hippuric acid after benzoate ingestion, and, as these authors have discussed, this rate also represents the maximum rate of benzoic acid conjugation with glycine because a few hours after benzoate ingestion the urinary excretion rate of the conjugate is identical to the rate of conjugate formation. Also in the following discussion the urinary excretion rates measured some hours after the ingestion of benzoic acid and methylbenzoic acid have been used to express the conjugation rates of the respective compounds.

When doses of benzoic acid in the range of $41 \mathrm{mmol}(5 \mathrm{~g})$ were ingested, only a few percent of the total conjugates formed was acyl glucuronide (16). In this study renal excretion of methylbenzoic acid conjugates seemed to decrease temporarily when a large dose of benzoic acid taken $2 \mathrm{~h}$ after the ingestion of methylbenzoic acid apparently saturated the glycine conjugating capacity.
Urinary excretion of total methylbenzoic acid conjugates increased again during the next $2 \mathrm{~h}$ in spite of the still maximal benzoic acid conjugation (fig. 2). Whether this phenomenon was associated with a proportionately greater glucuronidation is not known because specific analyses were not made, but it is a distinct possibility since glucuronidation is an alternative pathway of benzoate and methylbenzoate metabolism $(2,16)$. During the first $8 \mathrm{~h}$ after the ingestion of methylbenzoic acid there were frequently trace amounts of the free acid in the urine. Excretion rates of the free acid were highest (but at most only $0.32 \mu \mathrm{mol} / \mathrm{min}$ ) immediately after the large dose of benzoic acid (fig. 2). This finding could suggest some cumulation of methylbenzoic acid in the body although there is also the possibility that the free acid originated from the glucuronide, which, like benzoyl glucuronide (16), may relatively easily be broken down.

There was no interaction between the renal excretion of methylhippuric acid and a larger dose of hippuric acid (fig. 1); this finding is entirely consistent with the known large capacity of proximal tubular secretion for high $\mathrm{T}_{\mathrm{m}}$ organic acids such as hippurates and p-aminohippurates (11). Tubular secretion may be more easily interfered with by the concomitant ad- 
Table 2. Ionization characteristics (pKa) of some organic acids.

\begin{tabular}{lccc}
\hline \multirow{2}{*}{ Compound } & \multicolumn{3}{c}{ pKa from different sources } \\
\cline { 2 - 4 } & $\mathrm{C} / \mathrm{P}^{\mathrm{a}}$ & Lange $^{\mathrm{b}}$ & Own estimation \\
\hline Hippuric acid & 3.8 & 3.7 & 3.7 \\
Methylhippuric acid (m-isomer) & & & 2.9 \\
\hline
\end{tabular}

a Handbook of Chemistry and Physics (45th ed.), The Chemical Rubber Co., Cleveland, $\mathrm{OH} 1964$.

b Lange's Handbook of Chemistry (11th ed.), McGraw-Hill Book Co., New York, NY 1973.

c Courtesy of P. Pfäffli, M.Sc.

ministration of a low $T_{m}$ compound, such as probenecid (11). The full course of methylhippuric acid and, in particular, hippuric acid excretion after the ingestion of the conjugated acids was remarkably slower than that of the same compounds after the ingestion of the unconjugated acids. On the other hand, in the latter case the excretion rate of methylhippuric acid approached the corresponding value for injected methylhippuric acid.

Let us assume that the maximum capacity of glycine conjugation in a $70-\mathrm{kg}$ well-nourished man is $190 \mu \mathrm{mol} / \mathrm{min}$ and that this step is the rate-limiting one in toluene and xylene biotransformation. When a person inhales toluene or xylene, about $60 \%$ of the inhaled amount is retained in the body $(15,18)$. With this figure for retention and pulmonary ventilation of $10 \mathrm{l} / \mathrm{min}$, which is typical for light work, pulmonary uptake of toluene or xylene approaches the full metabolic capacity when the inhaled concentration is about $32 \mathrm{mmol} / \mathrm{m}^{3}$ (780 ppm). If the exposure involves moderately heavy work (pulmonary ventilation $30 \mathrm{l} / \mathrm{min}$ ), saturation of the metabolic capacity is reached at an inhaled concentration of $11 \mathrm{mmol} / \mathrm{m}^{3}$ $(270 \mathrm{ppm})$. These figures illustrate the fact that in actual occupational exposure to toluene and xylene, which may well constitute solvent air concentrations in the range of $100-200 \mathrm{ppm}$ and involve even heavy physical exercise, a remarkable share of the capacity to mobilize glycine is being occupied. To this level must still be added the metabolic requirement of the normal dietary intake of benzoates and quinic acid (14), which maintain a notable "base-line" level of renal hippurate excretion (fig. 3).
It should even be emphasized that the availability of glycine may be considerably less in old age, undernutrition and other pathophysiological conditions (6) and that other compounds such as drugs may compete for it. Because benzoates seem to be good substrates for glycine conjugation, the consequence may well be a disturbance in the metabolism of the competing compound. For instance, it was shown that saturation of glycine conjugation with benzoate hinders normal biotransformation and hence excretion of salicylic acid so that the drug may hazardously cumulate in the body $(1,8)$. Furthermore, it has been known for a long time that ingesting greater amounts of benzoic acid decreases the renal excretion of uric acid (14). Among a group of workers occupationally exposed to toluene and xylene, a similar and dose-dependent decrease in the urinary excretion of uric acid was noted (10). This phenomenon has been attributed to the consumption of a major part of the bodily glycine pool by benzoate and methylbenzoate metabolism $(10,14)$, but a more probable mechanism is the effect of organic acids in inhibiting urate secretion at low dosage levels (11).

Even though metabolic interactions may readily result from the limited availability of glycine, it can be recognized that the conjugation and excretion of the metabolic intermediates of toluene and xylene (benzoic acid and methylbenzoic acid) are likely to be guaranteed even at a relatively high level of occupational exposure. If, however, the full conjugating capacity is occupied, the excretion of toluene and xylene metabolites will inevitably be delayed. Because the tubular secretion capacity for hippurates is at least two times 
larger than the capacity for hippurate formation $(1,20)$, renal excretion, under conditions of normal kidney physiology, will not determine the excretion kinetics of toluene or xylene, contrary to what has been proposed (19).

Once hippuric acid and methylhippuric acid have been secreted into the proximal tubular fluid, their further excretion by the kidneys is probably fairly uncomplicated. Significant reabsorption is unlikely because these compounds are watersoluble and they occur mainly dissociated at normal urinary $\mathrm{pH}$ levels. The latter is particularly true of methylhippuric acid, while the somewhat higher pKa value for hippuric acid (table 2) indicates that a portion will be unionized in the more acid urine. Hippurate excretion is also insensitive to the variations in urine flow rate caused by the variable water intake (14) (fig. 3) characteristic of active proximal tubular transport (17). At exceptionally low rates of diuresis $<30$ $\mathrm{ml} / \mathrm{h}$ ), however, basal urinary excretion of hippurate has been shown to decrease (21).

\section{ACKNOWLEDGMENTS}

The author is greatly indebted to Ms. K. Pekari, M.Sc., who supervised all the analytical work.

\section{REFERENCES}

1. AMSEL, I. P. and LEVY, G. Drug biotransformation interactions in man: II. A pharmacokinetic study of the simultaneous conjugation of benzoic and salicylic acids with glycine. J. pharm. sci. 58 (1969) $321-326$

2. BRAY, H. G., HUMPHRIS, B. G. and THORPE, W. V. Metabolism of derivatives of toluene--3. o-, $\mathrm{m}$ - and p-xylenes. Biochem. j. 45 (1949) $241-244$.

3. BRIDGES, J. W., FRENCH, M. R., SMITH, R. L. and WILLIAMS, R. T. The fate of benzoic acid in various species. Biochem. $j$. 118 (1970) $47-51$
4. DETTLI, L. Pharmacokinetic aspects of drug interactions. In: L. E. CLUFF and J. C. PETRIE (eds.), Clinical effects of interaction between drugs. Excerpta Medica, Amsterdam 1974, pp. 39-68.

5. ENGSTRÖM, K., HUSMAN, K. and RANTANEN, J. Measurement of toluene and xylene metabolites by gas chromatography. Int. arch. occup. environ. health 36 (1976) $156-160$.

6. IRJALA, K. Synthesis of p-aminohippuric, hippuric and salicyluric acids in experimental animals and man. Ann. acad. sci. fenn. ser. A 5 (1972) 154.

7. LAHAM, S. Metabolism of industrial solvents. Ind. med. surg. 39 (1970) 61-64.

8. LEVY, G., AMSEL, L. P., ELLIOTT, H. C. Kinetics of salicyluric acid elimination in man. J. pharm. sci. 58 (1969) 827-829.

9. MIKULSKI, P. and WIGLUSZ, R. Comparison of benzene and its methyl derivatives in the rat and stimulatory effect of phenobarbital. Bull. inst. mar. med. gdańsk 23 (1972) 153-160.

10. MIKULSKI, P., WIGLUSZ, R., BUBLEWS$\mathrm{KA}$, A. and USELIS, J. Investigation of exposure of ship's painters to organic solvents. $B r . j$. ind. med. 29 (1972) $450-453$.

11. MILNE, M. D. Drug interactions and the kidney. In: L. E. CLUFF and J. C. PETRIE (eds.), Clinical effects of interaction between drugs. Excerpta Medica, Amsterdam 1974, pp. 193-228.

12. OGATA, M., TAKATSUKA, Y. and TOMOKUNI, K. Excretion of hippuric and $\mathrm{m}$ - or $\mathrm{p}$-methylhippuric acid in the urine of persons exposed to vapours of toluene and $\mathrm{m}$ - or $\mathrm{p}$-xylene in an exposure chamber and in workshops, with specific reference to repeated exposures. $B r . j$. ind. med. 28 (1971) 382-385.

13. OGATA, M., TOMOKUNI, $K$. and TAKATSUKA, Y. Urinary excretion of hippuric acid and $\mathrm{m}$ - or p-methylhippuric acid in the urine of persons exposed to vapours of toluene and $m$ - or $p$-xylene as a test of exposure. $B r . j$. ind. med. 27 (1970) $43-50$.

14. QUICK, A. J. The conjugation of benzoic acid in man. J. biol. chem. 92 (1931) 65 -85 .

15. RÖMMELT, $H$. and DIRNAGL, K. Pulmonale Resorption von sechs Kohlenwasserstoffen in Abhängigkeit von der Konzentration in der Atemluft. Münch. Med. Wochenschr. 119 (1977) 367-368.

16. SCHACHTER, D. The chemical estimation of acyl glucuronides and its application to studies on the metabolism of benzoate and salicylate in man. $J$. clin. invest. 36 (1957) $297-302$.

17. SCHÜCK, O., NADVORNIKOVA, H. and GRAFNETTEROVA, J. Clinical localization of tubular transport of exogenous substances exhibiting urine flow dependent excretion. Int. j. clin. pharmacol. 16 (1978) $307-309$.

18. SEDIVEC, V. and FLEK, J. The absorption, metabolism, and excretion of xylenes 
in man. Int. arch. occup. environ. health 37 (1976) 205-217.

19. SENCZUK, W. and ORLOWSKI, J. Absorption of $m$-xylene vapours through the respiratory tract and excretion of $m$-methylhippuric acid in urine. $\mathrm{Br}$. $j$. ind. med. 35 (1978) $50-55$.

Received for publication: 3 April 1979
20. WESSON, L. G. JR. Physiology of human kidney. Grune \& Stratton, New York, NY 1969 , p. 173.

21. WILCZOK, T. and BIENEK, G. Urinary hippuric acid concentration after occupational exposure to toluene. $B r . j$. ind. med. 35 (1978) $330-334$. 